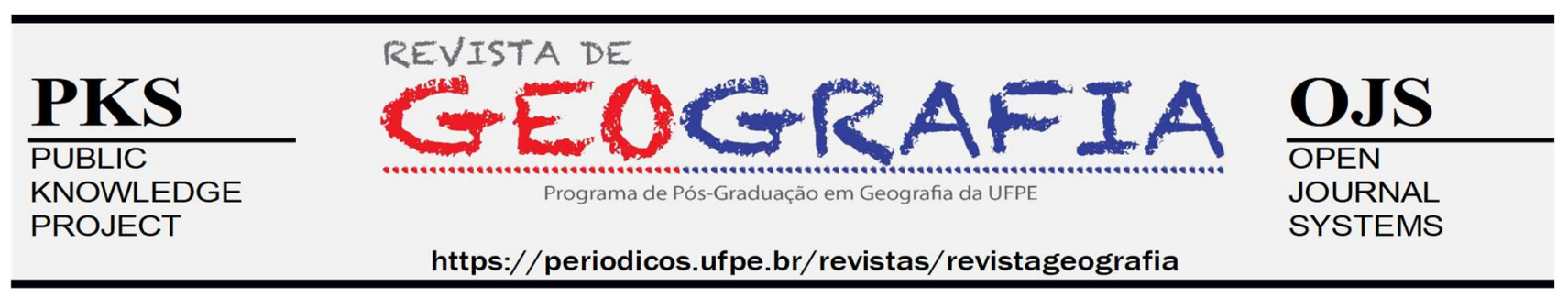

\title{
QUANTIFICAÇÃO DA PEGADA HÍDRICA DA SOJA NA REGIÃO DO MATOPIBA: CENÁRIO ATUAL E MUDANÇAS CLIMÁTICAS
}

\author{
Roberta Araújo e Silva ${ }^{1}$, Vicente de Paulo Rodrigues da Silva² ${ }^{2}$, Antonia Clemilda Nunes ${ }^{3}$, Pedro \\ Henrique da Conceição Silva ${ }^{4}$, Alessandra Maria Lima Naoe ${ }^{5}$ Girlene Figueiredo Maciel $^{6}$
}

\begin{abstract}
${ }^{1}$ Doutora Meteorologista da Universidade Federal do Tocantins (UFT) Campus Palmas, roberta.as@uft.edu.br (autor correspondente), https://orcid.org/0000-0002-9682-3006

${ }^{2}$ Doutor Professor Titular da Universidade Federal de Campina Grande (UFCG), vicente.paulo@dca.ufcg.edu.br, https://orcid.org/0000-0003-4914-4833

3 Doutora Engenheira Agrônoma, Universidade Federal do Tocantins (UFT) Campus Palmas, clemilda@mail.uft.edu.br,https://orcid.org/0000-0002-9682-3006

${ }^{4}$ Mestre Professor do Instituto Federal de Educação, Ciência e Tecnologia do Tocantins (IFTO) Campus Paraíso do Tocantins, pedro.silva@ifto.edu.br,https://orcid.org/0000-0002-1271-8269

${ }_{5}^{5}$ Doutora Engenheira Ambiental, Universidade Federal do Tocantins (UFT) Campus Palmas, alima@mail.uft.edu.br, https://orcid.org/0000-0002-1271-8269

${ }^{6}$ Doutor Professor Ajunto da Universidade Federal do Tocantins (UFT) Campus Palmas, maciel@mail.uft.edu.br, http://orcid.org/0000-0001-9217-3731
\end{abstract}

Artigo recebido em 05/05/2020 e aceito em 12/08/2020

\section{RESUMO}

A soja (Glycine max (L.) Merrill) é o principal cultivo agrícola produzido na região do Matopiba. Entretanto, o sucesso ou quebra da produção de soja está relacionada ao déficit e/ou excesso hídrico. A pegada hídrica (PH) avalia a pressão das atividades humanas sobre os recursos hídricos. Assim, esse artigo tem por objetivo investigar a produtividade e pegada hídrica da soja, sob cenário atual e mudanças climáticas. A produtividade e pegada hídrica futura foram obtidas através do modelo de crescimento de cultura AquaCrop da FAO e do downscaling dos modelos de circulação geral da atmosfera HadGEM2-ES e MIROC5, para os cenários de RCP 4.5 e 8.5. Atualmente a produtividade da soja na região do Matopiba varia de 2,6 a 3,0 t ha-1 e a $\mathrm{PH}_{\text {soja }}$ de 2.036,6 a 2.584,12 $\mathrm{m}^{3} \mathrm{t}^{-1}$. As projeções, geradas pelos modelos e RCP, sugerem aumento da produtividade da soja em até 2,0 tha ${ }^{-1}$ e decréscimo na $\mathrm{PH}_{\mathrm{soja}}$ de $700 \mathrm{~m}^{3} \mathrm{t}^{-1}$. A avaliação da $\mathrm{PH}_{\mathrm{soja}}$ futura, por suas componentes verde (água derivada da precipitação) e azul (de rios, subterrâneo), mostra que a demanda por água azul será maior que por água verde. A componente cinza (grau de poluição da água) da $\mathrm{PH}_{\text {soja }}$ diminui, mas esse componente deverá aumentar, uma vez que, considerou-se apenas fertilizantes nitrogenados e com valores praticamente constantes. Esses resultados ressaltam a importância de se criar estratégias de adequadas de manejo e cultivo, sob cenário de aumento produtividade de soja e da demanda por água azul, mantendo um uso aceitável dessa água.

Palavra-chave: Glycine max; AquaCrop; rendimento; clima futuro.

\section{QUANTIFICATION OF SOY WATER FOOTPRINT IN THE MATOPIBA REGION: CURRENT SCENARIO AND CLIMATE CHANGE}

\begin{abstract}
Soy (Glycine $\max (\mathrm{L}$.$) Merrill) is the main agricultural crop produced in the Matopiba region. However, the success$ or failure of soy production is available for this purpose and/or excess water. The water footprint $(\mathrm{PH})$ assessed the pressure of human activities on water resources. Thus, this article aims to investigate and track the water moisture in soybeans, in the current scenario and in climate change. A sample and future water index were affected by the growth model of FAO AquaCrop culture and the models of general circulation of the atmosphere HadGEM2ES and MIROC5 decreased, for the scenarios of RCP 4.5 and 8.5. Currently, the study of soy in the Matopiba region ranges from 2.6 to $3.0 \mathrm{t} \mathrm{ha}^{-1}$ and $\mathrm{PH}$ from 2,036.6 to 2,584.12 $\mathrm{m}^{3} \mathrm{t}^{-1}$. The evaluation of the future $\mathrm{PH}_{\text {soja }}$, by components its green (water derived from precipitation) and blue (river, underground), shows that the demand for
\end{abstract}


blue water will be greater than for green water. The gray component (degree of water pollution) of $\mathrm{PH}_{\text {soja }}$ decreases, but this component should increase, since it was considered only nitrogen fertilizers and with practically constant values. These results highlight the importance of creating appropriate management and cultivation strategies, under a scenario of increased soybean productivity and the demand for blue water, maintaining an acceptable use of this water.

Keyword: Glycine max; AquaCrop; Yield; Future climate.

\section{INTRODUÇÃO}

A soja (Glycine $\max (\mathrm{L}$.$) Merrill) é o principal cultivo agrícola produzido na região do$ Matopiba. Na Safra 2016/2017 a soja produzida nessa região superou 20 mil toneladas, em uma área plantada de 7.322 mil hectares. As projeções preveem incremento tanto na produção e na área plantada de soja. Entretanto, o sucesso ou quebra de produção desta cultura está relacionada ao déficit e/ou excesso hídrico, como também ao aumento da temperatura (Souza et al., 2016). Segundo Rolla et al. (2018), a agricultura, sob cenários de mudanças climáticas, aumentará o impacto sobre os recursos hídricos, devido a maior demanda por água e maior uso de pesticidas. A comunidade científica visando minimizar os efeitos da agricultura sobre a água tem buscado reformulações das atuais práticas agrícolas e ambientais, aliando estratégias que conduzam ao uso sustentável dos recursos hídricos pela agricultura. Um conceito chave da quantificação de água virtual é o paradigma para a avaliação do uso da água na produção de bens e especialmente comida, denominado por Pegada Hídrica (PH) (Hoekstra, 2003). A PH fornece cálculos cumulativos da pressão que as atividades humanas, ao longo de todo o processo (produto/serviço), exercem sobre os recursos hídricos, considerando os usos diretos e indiretos durante toda a cadeia produtiva. A quantificação da PH da cultura é benéfica para a avaliação do uso da água, melhorando a eficiência do seu uso, diminuindo o volume do consumo de água pela agricultura (Hoekstra e Hung, 2002; Hoekstra, 2003).

A intensificação do crescimento da produção de soja observada nas últimas décadas na região do Matopiba, provavelmente, implicará em aumento da pressão sobre os recursos hídricos da região. Sob cenários de mudanças climáticas a sustentabilidade desse recurso poderá ser afetada, à longo prazo. No contexto da importância econômica da soja para a região do Matopiba, da sua demanda hídrica e a influência das intempéries sobre o seu rendimento, a presente pesquisa tem por objetivo calcular a produtividade e a pegada hídrica da cultivar de soja TMG 1288 RR, contabilizando a pressão das atuais práticas agrícolas sobre os recursos hídricos da região e mediante as mudanças climáticas. 


\section{MATERIAL E MÉTODOS}

A região do Matopiba compreende todo estado do Tocantins, sul e nordeste do Maranhão, sul do Piauí e oeste da Bahia, regiões onde predomina a agricultura de sequeiro. São observadas três zonas climáticas: a tropical Brasil central (53\%), a tropical zona equatorial (44\%) e a equatorial (3\%). $\mathrm{Na}$ extensão central, o clima tropical semiúmido é dominante e corresponde a aproximadamente $78 \%$ da região, tendo como característica períodos de 7 a 8 meses sem precipitação e temperatura do ar média acima de $18^{\circ} \mathrm{C}$ em todos os meses do ano. No limite leste, tem-se clima semiárido, com temperaturas elevadas e período de 6 meses sem precipitação.

A determinação da pegada hídrica da soja $\left(\mathrm{PH}_{\text {soja }}\right)$, foi calculada como Hoekstra e Chapagain (2008) e Hoekstra et al. (2011) para a contabilização da pegada hídrica de uma cultura e em estudos sobre uso de água de agricultura por Rost et al. (2008) e Fader et al. (2011). A PH de uma cultura consiste na soma das suas componentes verde, azul e cinza do processo de crescimento da cultura e é calculada para cada componente dividida pela produtividade da cultura. Assim, a PH expressa a quantidade de água envolvida dentro da produção da cultura, representando a quantidade de água, em $\mathrm{m}^{3}$, necessária para produzir uma tonelada da produção colhida.

$$
\mathrm{PH}_{\text {soja }}=\mathrm{PH}_{\text {verde,soja }}+\mathrm{PH}_{\text {azul,soja }}+\mathrm{PH}_{\text {cinza,soja }}
$$

em que:

$\mathrm{PH}_{\text {soja }}=$ Pegada hídrica da cultura da soja $\left(\mathrm{m}^{3} \mathrm{t}^{-1}\right)$;

$\mathrm{PH}_{\mathrm{verde}, \mathrm{soja}}=$ Pegada hídrica verde da cultura da soja $\left(\mathrm{m}^{3} \mathrm{t}^{-1}\right)$;

$\mathrm{PH}_{\mathrm{azul}, \mathrm{soja}}=$ Pegada hídrica azul da cultura da soja $\left(\mathrm{m}^{3} \mathrm{t}^{-1}\right)$;

$\mathrm{PH}_{\text {cinza,soja }}=$ Pegada hídrica cinza da cultura da soja $\left(\mathrm{m}^{3} \mathrm{t}^{-1}\right)$.

A $\mathrm{PH}_{\mathrm{verde}, \text { soja }}$ refere-se ao consumo de água armazenada no solo como resultado da precipitação, a água que infiltra no solo e permanece por períodos curtos. É obtida pela razão entre a demanda de água verde da cultura da soja $\left(\mathrm{DHC}_{\mathrm{verde,soja}}\right)$ pela produtividade da cultura da soja (Prvt).

$$
\mathrm{PH}_{\text {verde,soja }}=\frac{\mathrm{DHC}_{\text {verde,soja }}}{\text { Prvt }}
$$

em que:

$\mathrm{PH}_{\text {soja,verde }}=$ Pegada hídrica verde da cultura da soja $\left(\mathrm{m}^{3} \mathrm{t}^{-1}\right)$;

$\mathrm{DCH}_{\text {verde,soja }}=$ Demanda de água verde da cultura da soja $\left(\mathrm{m}^{3} \mathrm{ha}^{-1}\right)$;

Prvt $=$ Produtividade da cultura da soja $\left(\mathrm{t} \mathrm{ha}^{-1}\right)$.

A $\mathrm{DHC}_{\mathrm{verde}, \mathrm{soja}}$ correspondendo ao total de água da chuva que sofre o processo de evapotranspiração. Calculado através do somatório do volume de água da precipitação, que é 
evapotranspirada diariamente pela cultura $\left(\mathrm{ET}_{\text {verde }}\right)$, desde o primeiro dia de plantio $(\mathrm{d}=1)$ até o fim da colheita (pdc).

$$
\mathrm{DHC}_{\text {verdesoja }}=10 \cdot \sum_{\mathrm{d}=1}^{\mathrm{pdc}} \mathrm{ET}_{\text {verde }}
$$

em que:

$\mathrm{DHC}_{\text {verde,soja }}=$ Demanda de água verde da cultura da soja $\left(\mathrm{m}^{3} \mathrm{ha}^{-1}\right)$;

10 é o fator de conversão, que visa converter a lâmina de água proveniente da chuva em milímetros para litros de água por superfície terrestre, $\mathrm{em} \mathrm{m}^{3} \mathrm{ha}^{-1}$;

$\mathrm{ET}_{\text {verde }}=$ Evapotranspiração diária de água verde $\left(\mathrm{mm} \mathrm{dia}^{-1}\right)$.

A evapotranspiração diária de água verde $\left(\mathrm{ET}_{\text {verde }}\right)$ é obtida a partir do mínimo entre os valores da precipitação efetiva (Pref) e evapotranspiração total da cultura $\left(\mathrm{ET}_{\mathrm{c}}\right)$. Neste trabalho foi obtida através do produto entre a evapotranspiração de referência $\left(\mathrm{ET}_{\mathrm{o}}\right)$ e o coeficiente de cultivo $\left(\mathrm{K}_{\mathrm{c}}\right)$.

A $\mathrm{PH}_{\text {azul,soja }}$ é a água que tem origem nos rios e lagos, ou extraída do subterrâneo. A componente azul da PH é obtida pela razão entre a demanda de água azul da cultura da soja $\left(\mathrm{DHC}_{\mathrm{azul}, \text { soja }}\right)$ e a produtividade dessa cultura da soja (Prvt) conforme a Eq. 4.

$$
\mathrm{PH}_{\text {azul,soja }}=\frac{\mathrm{DHC}_{\mathrm{azul}, \mathrm{soja}}}{\operatorname{Prvt}}
$$

A $\operatorname{DHC}_{\text {azulsoja }}$ (Eq. 5) é calculada através do somatório do volume de água azul, que é evapotranspirada diariamente pela cultura $\left(\mathrm{ET}_{\mathrm{azul}}, \mathrm{m}^{3} \mathrm{ou} \mathrm{L}\right)$, desde o primeiro dia de plantio $(\mathrm{d}=1)$ ao fim da colheita (pdc).

$$
\mathrm{DHC}_{\mathrm{azul}, \mathrm{soja}}=10 \cdot \sum_{\mathrm{d}=1}^{\mathrm{pdc}} \mathrm{ET}_{\mathrm{azul}}
$$

em que:

$\mathrm{DHC}_{\mathrm{azul}, \mathrm{soja}}=$ Demanda de água azul da cultura da soja $\left(\mathrm{m}^{3}\right)$;

10 é o fator de conversão, que visa converter a lâmina de água irrigada de milímetros para litros de água por superfície terrestre, em $\mathrm{m} 3 / \mathrm{ha}$;

$\mathrm{ET}_{\mathrm{azul}}=$ Evapotranspiração diária de água azul ( $\left.\mathrm{mm} / \mathrm{dia}\right)$.

$\mathrm{A} \mathrm{ET}_{\mathrm{azul}}$ é calculada a partir do máximo entre a diferença da evapotranspiração total da cultura (ETc), obtida através do produto entre a evapotranspiração de referência $\left(E_{0}\right)$ e o coeficiente de cultivo $\left(\mathrm{K}_{\mathrm{c}}\right)$, e a precipitação efetiva (Pref)

A pegada hídrica cinza $\left(\mathrm{PH}_{\text {cinza,soja }}\right)$ é um indicador do grau de poluição da água doce. Segundo Hoekstra et al. (2011), e é obtida através do produto entre a taxa de aplicação por hectare 
dos agroquímicos no campo (TAQ), e a fração de lixiviação/escoamento ( $\alpha$ ), dividido pela diferença entre a máxima concentração aceitável ( $\left.\mathrm{c}_{\max }\right)$ e a concentração natural do poluente em questão ( $\left.\mathrm{c}_{\mathrm{nat}}\right)$, divididas então pela produtividade da cultura da soja (Prtv), como mostra a Eq. 6.

$\mathrm{PH}_{\text {cinza,soja }}=\frac{\frac{(\alpha \cdot \text { TAQ })}{\left(\mathrm{C}_{\max }-\mathrm{C}_{\text {nat }}\right)}}{\operatorname{Prvt}}$

em que:

$\alpha=$ fração de lixiviação/escoamento do fertilizante;

$\mathrm{TAQ}=$ taxa de aplicação por hectare do composto agroquímico em campo $\mathrm{kg} \mathrm{ha}^{-1}$;

$\mathrm{C}_{\max }=$ concentração máxima aceitável de poluentes no sistema $\left(\mathrm{t} \mathrm{m}^{-3}\right)$;

$\mathrm{C}_{\text {nat }}=$ concentração natural num corpo de água $\left(\mathrm{t} \mathrm{m}^{-3}\right)$. No caso de concentrações naturais não conhecidas com precisão, mas estimativas que sejam baixas, admite-se que a concentração natural da massa de água receptora é nula $\left(\mathrm{C}_{\mathrm{nat}}=0\right)$;

Prvt $=$ produtividade da cultura da soja $\left(\mathrm{t} \mathrm{ha}^{-1}\right)$.

A fonte de poluição adotada foi o fertilizante nitrogenado. Segundo Lamond e Wesley (2001), a aplicação de nitrogênio recomendada é de $22 \mathrm{~kg} \mathrm{ha}^{-1}$. Seguindo o padrão da Resolução CONAMA 357/2005, classe 3 para águas doces que é de $10 \mathrm{mg} \mathrm{L}^{-1}$. Atribuiu-se o valor 0 (zero) para a concentração natural do nitrogênio no corpo hídrico. A fração de lixiviação adotada foi de $10 \%$ (Chapagain et al., 2006).

A produtividade atual da cultura da soja foi obtida junto ao Instituto Brasileiro de Geografia e Estatística (IBGE) e as produtividades futuras foram estimadas por meio do modelo AquaCrop, usando os parâmetros de solo da região, as séries de dados meteorológicos dos modelos de circulação geral da atmosfera (MCGA) e a calibração do modelo AquaCrop para a cultura soja na região do Matopiba obtida por Silva et al. (2018).

Os dados meteorológicos necessários para a geração dos cenários de mudanças climáticas foram obtidos considerando os MCGA: HadGEM2-ES, modelo britânico da Met Office Hadley Center, com resolução de $1,875^{\circ}$ x $1,25^{\circ}$ (Bellouin et al., 2011); e o japonês MIROC5 da Center for Climate System Research - CCSR; University of Tokyo; National Institute for Environmental Studies - NIES; Japan Agenc for Marine-Earth Science and Technology; com resolução de $1,4^{\circ}$ x $1,6^{\circ}$ (Watanabe et al., 2011). A fim de se obter maior representatividade espacial e temporal dos dados meteorológicos gerados pelos GCMs, utilizou-se o como downscaling o MarkSimGCM (http://gisweb.ciat.cgiar.org/MarkSimGCM/), que é um gerador de dados meteorológicos, que pode 
ser usado por qualquer modelo agrícola (Jones e Thornton, 2013; Tongson et al., 2017; Habtemariam et al.; 2017).

A evapotranspiração atual e futura para a região do Matopiba foi obtida através do método de Hagreaves (Hagreaves e Samani, 1985), a partir das séries diárias de temperatura do ar máxima e mínima geradas pelo MarkSimGCM. Os cenários de emissões de gases estufa adotados foram as Trajetórias de Representatividade de Concentração - RCP (Representative Concentration Pathaway), do Quinto Relatório de Avaliação do IPCC - AR5 (2014). As RCP selecionadas foram as RCP 4.5 e RCP 8.5, com e sem estratégias de adaptação no futuro, respectivamente. As produtividades foram geradas para cada modelo, cenário e períodos futuros a médio (2045/2046 e 2055/2056) e a longo prazo (2075/2076 e 2094/2095). Como na região do Matopiba nem todos os municípios são produtores de soja, selecionou-se apenas o maior produtor de soja de cada estado que compõem o Matopiba (Figura 1), são eles: Campos Lindos - TO, Formosa do Rio Preto - BA, Baixa Grande do Ribeiro - PI e Balsas - MA. A semeadura considerada foi de 01 de novembro para todos os municípios e períodos, representando a época de plantio de grãos característica da região do Matopiba.

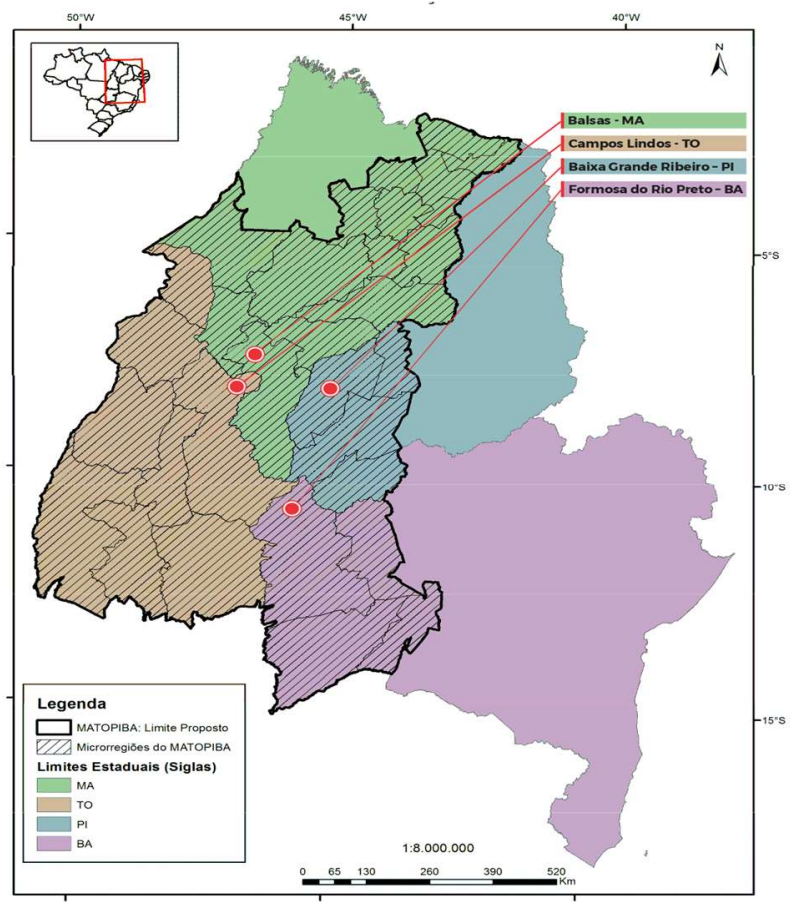

Figura 1. Localização da região do Matopiba e dos principais municípios produtores de soja, de cada um dos quatro estados que compõem a região. Fonte: EMBRAPA, 2014, modificado pelo autor. 


\section{RESULTADOS E DISCUSSÃO}

As simulações de temperatura do ar, para a região do Matopiba, mostradas na Figura 2, sugere incremento em ambos os modelos e cenários de RCP. As projeções para Balsas - MA e Formosa do Rio Preto - BA, sob a RCP 4.5, são de incremento de 3,0 ${ }^{\circ} \mathrm{C}$ para o HadGEM2-ES e de 2,5 $5^{\circ}$ a 2,9 $9^{\circ}$ p para o MIROC5. Em Campos Lindos - TO e Baixa Grande do Ribeiro - PI, sob a RCP 4.5, o incremento varia de $2,2^{\circ} \mathrm{C}$ a $2,8^{\circ} \mathrm{C}$ para o HadGEM2-ES e de $1,85^{\circ} \mathrm{C}$ a $1,91^{\circ} \mathrm{C}$ para o MIROC5. Sob o cenário de RCP 8.5, as projeções sugerem incremento mais acentuado. Em Balsas MA o incremento é de apenas $1,6^{\circ} \mathrm{C}$, para o HadGEM2-ES, porem para o MIROC5 o aumento pode chegar a 5,30 C. Em Campos Lindos - TO, verifica-se situação posta, o maior incremento foi obtido pelo HadGEM2-ES, de 5,7 ${ }^{\circ}$, e para o MIROC5, de 5,1 ${ }^{\circ}$ C. Em Baixa Grande do Ribeiro - PI, para ambos os modelos e cenários de RCP, o aumento será de $5^{\circ} \mathrm{C}$. Formosa do Rio Preto - BA é o que apresenta maior incremento de temperatura, de $6^{\circ} \mathrm{C}$, em ambos os modelos e cenários de RCP.

a)

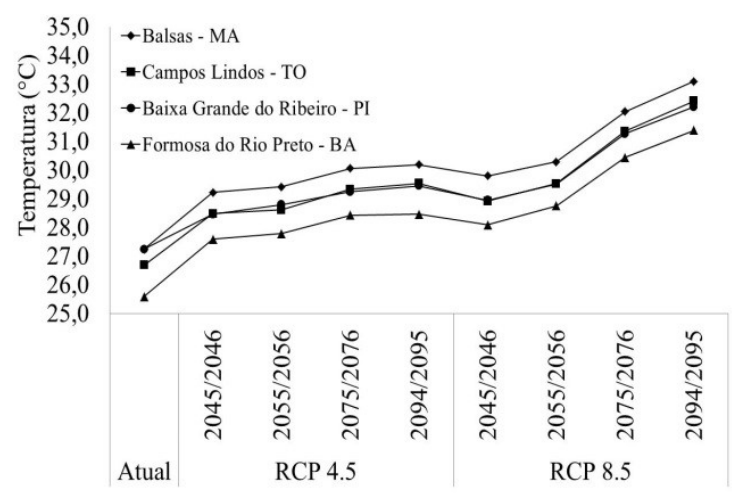

b)

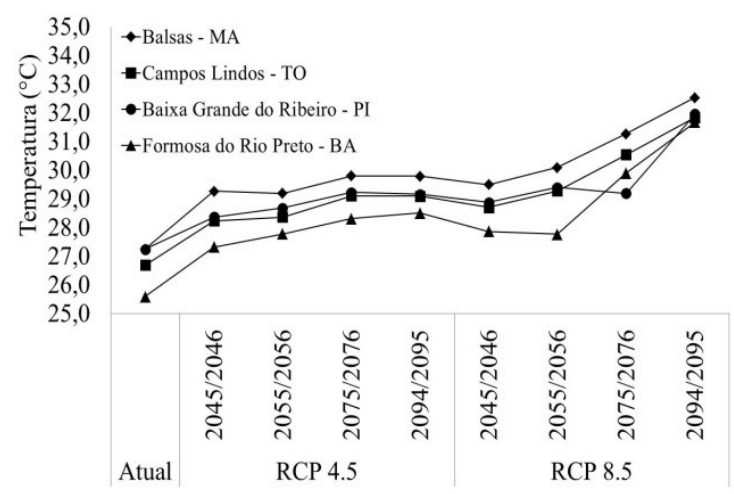

Figura 2. Temperatura média do ar durante o ciclo de crescimento da soja, entre 01 de novembro e 10 de março, projetada pelos modelos HadGEM2-ES (a) e MIROC5 (b), sob os cenários de RCP 4.5 e RCP 8.5. Para os três períodos: atual (referência), médio prazo (2045/2046 e 2055/2056) e longo prazo (2075/2076 e 2094/2095) para os municípios de Balsas - MA, Campos Lindos - TO, Baixa Grande do Ribeiro - PI, e Formosa do Rio Preto - BA.

As precipitações projetadas pelos modelos HadGEM2-ES e MIROC5, apresentam grande variação espaço-temporal (Figura 03). Comparando os totais de precipitação atual com as projeções geradas pelo HadGEM2-ES e sob a RCP 4.5, verifica-se para o período a médio prazo (2045/2046 a 2055/2056), aumento nos acumulados de precipitação para todos os quatro municípios. 
a)

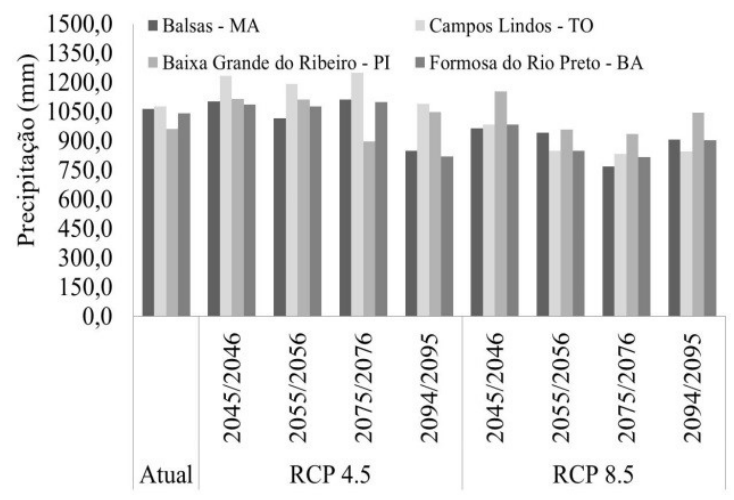

b)

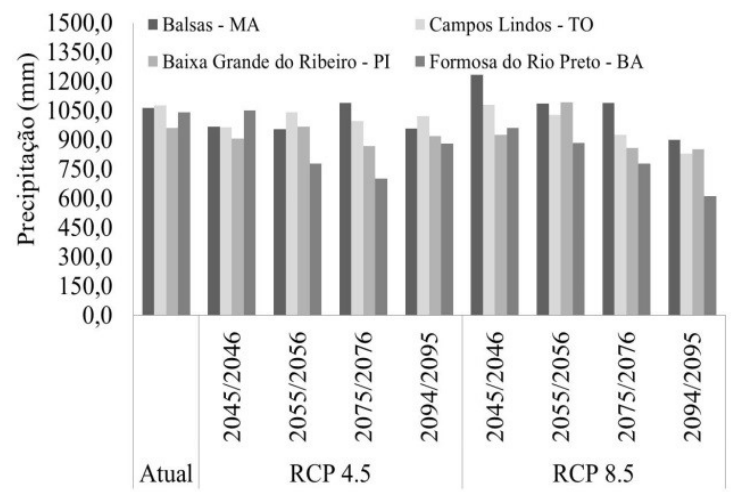

Figura 3. Precipitação acumulada durante o ciclo de crescimento da soja, entre 01 de novembro e 10 de março, projetada pelos modelos HadGEM2-ES (a) e MIROC5 (b), sob os cenários de RCP 4.5 e RCP 8.5. Para os três períodos: atual (referência), médio prazo (2045/2046 e 2055/2056) e longo prazo (2075/2076 e 2094/2095) para os municípios de Balsas - MA, Campos Lindos - TO, Baixa Grande do Ribeiro - PI, e Formosa do Rio Preto -BA.

Já, a longo prazo (2075/76 a 2094/2095), em Balsas - MA e Formosa do Rio Preto - BA a precipitação decresce em até 200,0 mm; em Campos Lindos - TO e Baixa Grande do Ribeiro - PI verifica-se aumento de até 173,0 mm. Para o cenário de RCP 8.5, o modelo HadGEM2-ES projeta decréscimo tanto a médio, quanto a longo prazo em quase todos os municípios, a exceção de Baixa Grande do Ribeiro - PI que apresenta aumento de até 194,0 mm. Nas projeções geradas pelo MIROC5, sob as RCP 4.5 e 8.5, predomina decréscimo de precipitação para todos os municípios e períodos (médio e longo prazo), variando em intensidade ao longo do tempo. Destacando-se as projeções decréscimo em Formosa do Rio Preto - BA, de até 342,0 mm, sob a RCP 4.5, e de 431,0 mm, sob a RCP 8.5. A concentração atual e futura de $\mathrm{CO}_{2}$ na atmosfera, adotados neste estudo, são mostradas Tabela 1. A média atual de $\mathrm{CO}_{2}$ na atmosfera é de $378,8 \mathrm{ppm}$. As projeções, sob a RCP 4.5, são de incremento de 96,2 ppm e 157,2 ppm, respectivamente, para o período a médio e longo prazo. Para o cenário de RCP 8.5, a concentração de $\mathrm{CO}_{2}$ é mais acentuada, praticamente dobra, com aumento de 137,2 ppm no período a médio prazo e de 506,8 ppm a longo prazo.

Tabela 1. Concentração de $\mathrm{CO}_{2}$ para o cenário atual e sob os cenários de RCP 4.5 e RCP 8.5.

\begin{tabular}{|c|c|c|c|c|c|}
\hline \multirow{3}{*}{$\begin{array}{c}\mathrm{CO}_{2}(\mathrm{ppm}) \\
\text { Cenário }\end{array}$} & \multicolumn{5}{|c|}{ Período } \\
\hline & \multirow[t]{2}{*}{ Atual } & \multicolumn{2}{|c|}{ Médio prazo } & \multicolumn{2}{|c|}{ Longo prazo } \\
\hline & & $2045 / 2046$ & $2055 / 2056$ & $2075 / 76$ & $2094 / 2095$ \\
\hline $\begin{array}{l}\text { RCP } 4.5 \\
\text { RCP } 8.5\end{array}$ & 378,8 & $\begin{array}{l}475,0 \\
516,0\end{array}$ & $\begin{array}{l}500,0 \\
575,0\end{array}$ & $\begin{array}{l}530,0 \\
720,0\end{array}$ & $\begin{array}{l}536,0 \\
885,0\end{array}$ \\
\hline
\end{tabular}


A produtividade atual e futura da soja, estimadas para os municípios que representam a região do Matopiba, geradas pelos modelos HadGEM2-ES e MIROC5, para os cenários de RCP 4.5 e 8.5, são mostradas na Figura 4. A produtividade média atual na região do Matopiba é de $2,83 \mathrm{t} \mathrm{ha}^{-}$ 1, sendo que a maior produtividade é encontrada para Campos Lindos - TO $\left(3,07 \mathrm{t} \mathrm{ha}^{-1}\right)$ e a menor para Baixa Grande do Ribeiro - PI (2,67 t ha-1).

a)

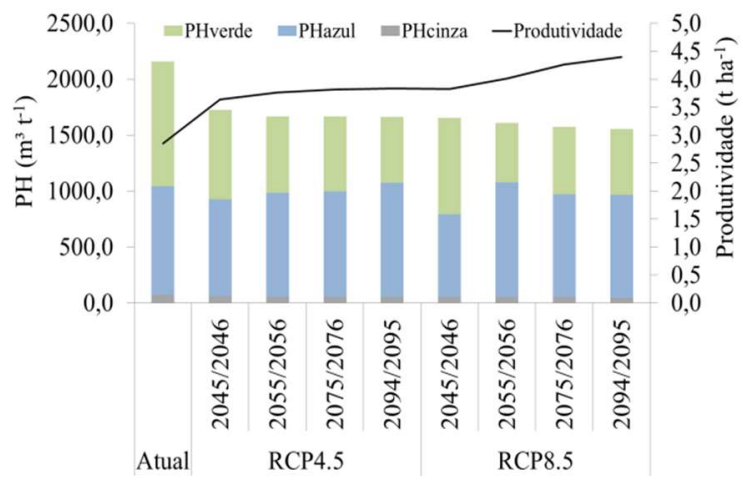

c)

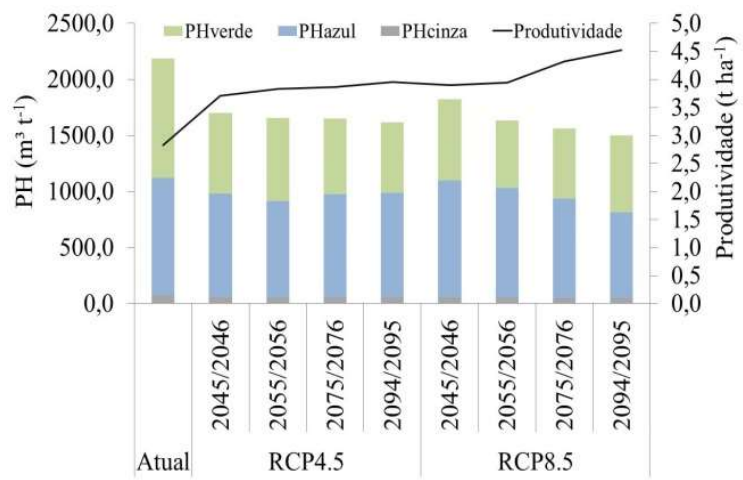

b)

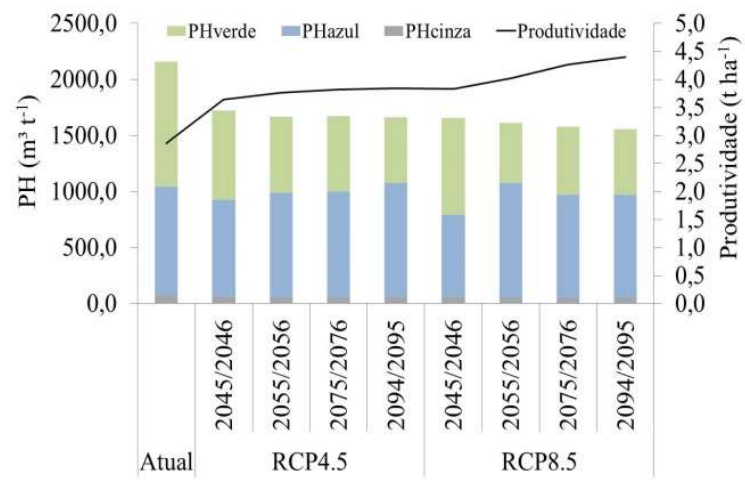

d)

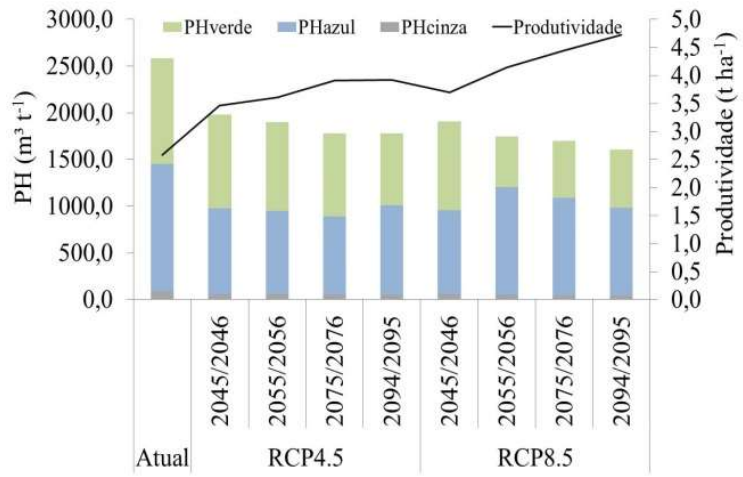

Figura 4. Estimativa da $\mathrm{PH}_{\text {soja }}$ verde, azul e cinza (colunas) e a produtividade total (linhas) para a cultivar de soja TMG 1288RR, projetada pelos modelos HadGEM2-ES para o cenário de RCP 4.5 e RCP 8.5, para os três períodos: atual (referência), médio prazo (2045/2046 e 2055/2056) e longo prazo (2075/2076 e 2094/2095), para os municípios de Balsas - MA (a), Campos Lindos - TO (b), Baixa Grande do Ribeiro - PI (c) e Formosa do Rio Preto -BA (d).

A produtividade estimada pelo HadGEM2-ES, sob a RCP 4.5, deve aumentar de 3,0 a 3,8 t ha $^{-1}$ em Balsas - MA (Figura 4a) e Campos Lindos - TO ( Figura 4b); e de 3,7 a 3,9 t ha ${ }^{-1}$ em Baixa Grande do Ribeiro - PI (Figura 4c); e de 3,5 a 3,9 t ha ${ }^{-1}$ em Formosa do Rio Preto - BA (Figura 4d). Para o HadGEM2-ES, sob a RCP 8.5, as produtividades apresentam incremento maior, com valores 
variando de: 3,8 a 4,4 $\mathrm{t} \mathrm{ha}^{-1}$ em Balsas - MA; 3,7 a 3,9 $\mathrm{t} \mathrm{ha}^{-1}$ em Campos Lindos - TO; 3,9 a 4,5 $\mathrm{t}$ ha ${ }^{1}$ em Baixa Grande do Ribeiro - PI; e de 3,7 a 4,7 t ha ${ }^{-1}$ em Formosa do Rio Preto - BA.

O MIROC5, sob a RCP 4.5, assinala aumento nas produtividades de 0,7 a 1,4 $\mathrm{t} \mathrm{ha}^{-1}$, com valores variando de: 3,5 a 3,9 t ha $\mathrm{t}^{-1}$ em Balsas - MA e Campos Lindos - TO; e de 3,7 a 4,0 t ha ${ }^{-1} \mathrm{em}$ Baixa Grande do Ribeiro - PI e Formosa do Rio Preto - BA. Para o cenário de RCP 8.5, o MIROC5 estimou maior incremento na produtividade da soja, de 0,8 a 2,0 $\mathrm{t} \mathrm{ha}^{-1}$, com valores variando de: 3,9

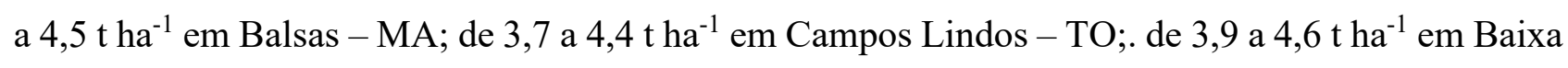
Grande do Ribeiro - PI; e de 3,6 a 4,8 em Formosa do Rio Preto - BA.

A $\mathrm{PH}_{\text {soja }}$ e a repartição em componentes (azul, verde e cinza), para os cenários atual e de mudanças climáticas (RCP 4.5 e 8.5), geradas através do modelo HadGEM2-ES para os quatro

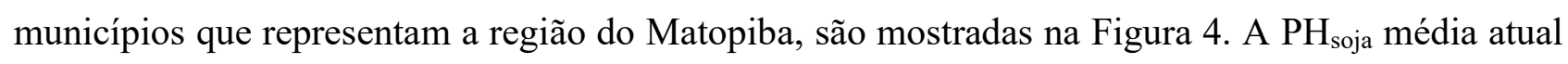
da na região do Matopiba é de 2241,83 $\mathrm{m}^{3} \mathrm{t}^{-1}$, com valores variando entre mínimo de 2036,6 $\mathrm{m}^{3} \mathrm{t}^{-1}$ (Balsas - MA) e máximo de 2584, $1 \mathrm{~m}^{3} \mathrm{t}^{-1}$ (Formosa do Rio Preto - BA). As componentes da $\mathrm{PH}_{\text {soja }}$ apresentam comportamento distinto em cada município (Tabela 2). Nos municípios de Balsas - MA e Formosa do Rio Preto - BA a maior contribuição percentual é da componente azul, respondendo respectivamente por 49,5\% e 52,8\%. Já nos municípios de Campos Lindos - TO e Baixa Grande do Ribeiro - PI é a componente azul é que responde pela maior contribuição percentual, de respectivamente $51,6 \%$ e 48,7\%. Quanto a componente cinza, a contribuição atual é pequena em todos os municípios. As projeções sugerem incremento na produtividade da soja em todos os modelos e cenários climáticos.

Nas projeções de $\mathrm{PH}_{\text {soja, }}$ geradas pelo modelo HadGEM2-ES, para todos os municípios que representam a região do Matopiba, verifica-se decréscimo da $\mathrm{PH}_{\text {soja }}$ a médio e longo prazo, em ambos cenários de RCP (Figura 4). No cenário de RCP 4.5 a $\mathrm{PH}_{\text {soja }}$ decresce em 374,4 $\mathrm{m}^{3}$ ton $^{-1}$ em Balsas MA (Figura 4a), 494,1 $\mathrm{m}^{3}$ ton $^{-1}$ em Campos Lindos - TO (Figura 4b), 572,4 $\mathrm{m}^{3}$ ton $^{-1} \mathrm{em}$ Baixa Grande do Ribeiro - PI (Figura 4c), e 800,6 $\mathrm{m}^{3}$ ton $^{-1}$ em Formosa do Rio Preto - BA (Figura 4d). Sob o cenário de RCP 8.5, esse decréscimo é mais acentuado, de 490,6 $\mathrm{m}^{3}$ ton $^{-1} \mathrm{em}$ Balsas - MA, 600,1 $\mathrm{m}^{3}$ ton $^{-1}$ em Campos Lindos - TO, 689,7 $\mathrm{m}^{3}$ ton $^{-1}$ em Baixa Grande do Ribeiro - PI, e 977,6 $\mathrm{m}^{3}$ ton $^{-1} \mathrm{em}$ Formosa do Rio Preto - BA. 
Tabela 2. Contribuição percentual das componentes verde, azul e cinza da $\mathrm{PH}_{\text {soja, }}$ atual (referência) e projetada pelo modelo HadGEM2-ES, para os cenários de RCP 4.5 e 8.5 e os período a médio prazo (2045/2046 e 2055/2056) e longo prazo (2075/2076 e 2094/2095), para os municípios de Balsas MA, Campos Lindos- TO, Baixa Grande do Ribeiro - PI e Formosa do Rio Preto -BA.

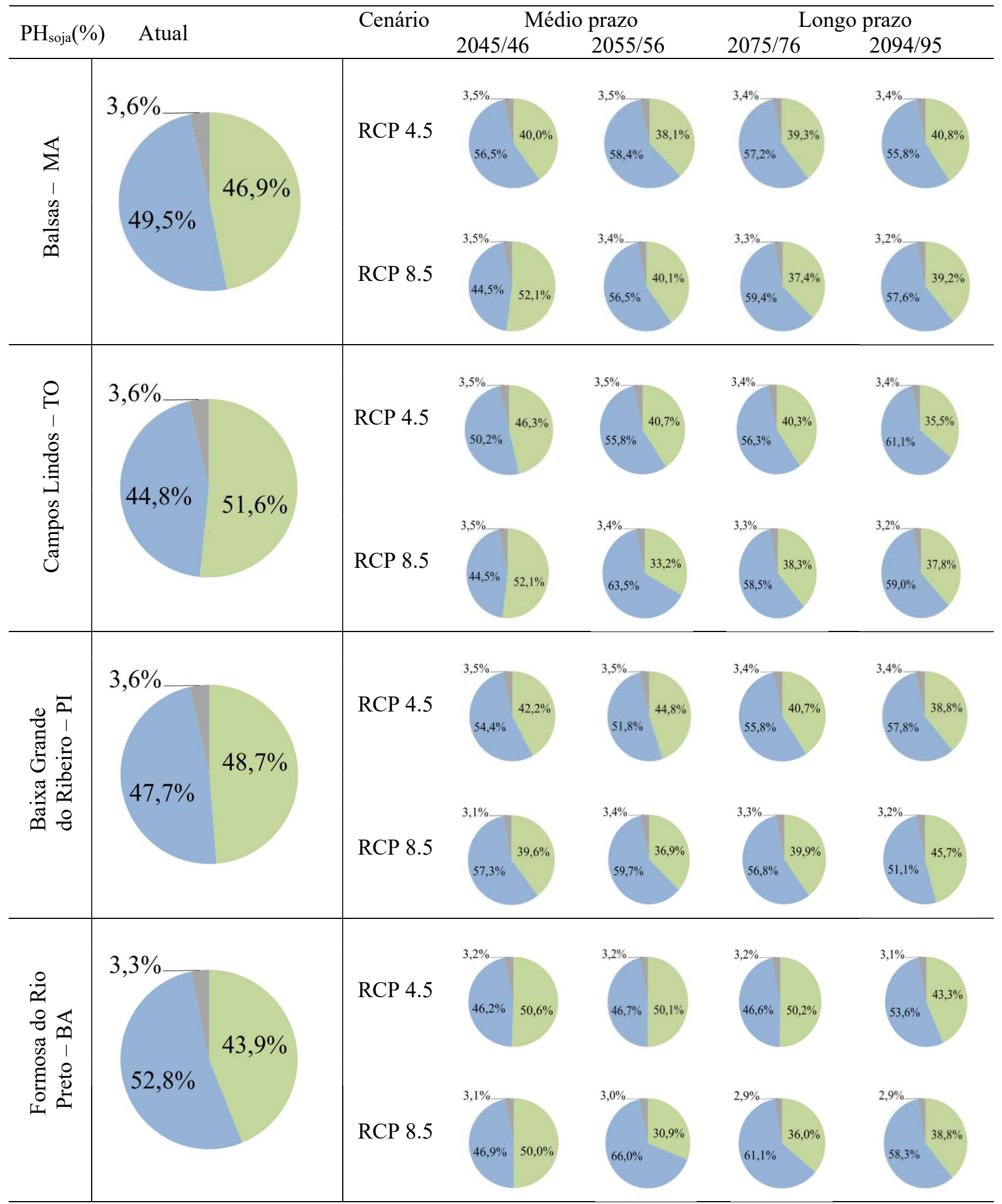


O modelo MIROC5 (Figura 5), para ambos os cenários de RCP, também assinala decréscimo da $\mathrm{PH}_{\text {soja. }}$ No cenário de $\mathrm{RCP} 4.5$, a $\mathrm{PH}_{\text {soja }}$ decresce $311,2 \mathrm{~m}^{3}$ ton $^{-1}$ em Balsas - MA (Figura 5a); 478,0 $\mathrm{m}^{3}$ ton $^{-1}$ Campos Lindos - TO (Figura 5b); 508,4 $\mathrm{m}^{3}$ ton $^{-1}$ em Baixa Grande do Ribeiro - PI (Figura 5c), e de 675,2 $\mathrm{m}^{3}$ ton $^{-1}$ em Formosa do Rio Preto - BA (Figura 5d). Sob o cenário de RCP 8.5, o decréscimo da $\mathrm{PH}_{\mathrm{soja}}$ é mais acentuado, de 481,2 $\mathrm{m}^{3}$ ton $^{-1} \mathrm{em}$ Balsas - MA, 574,1 $\mathrm{m}^{3}$ ton $^{-1}$ em Campos Lindos - TO, 639,4 $\mathrm{m}^{3}$ ton $^{-1}$ em Baixa Grande do Ribeiro PI, e 863,2 $\mathrm{m}^{3}$ ton $^{-1}$ em Formosa do Rio Preto - BA. As projeções para ambos os modelos e

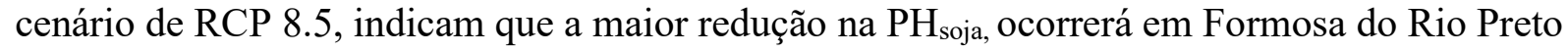
- BA, seguido por uma redução pouco menor em Balsas - MA.

a)

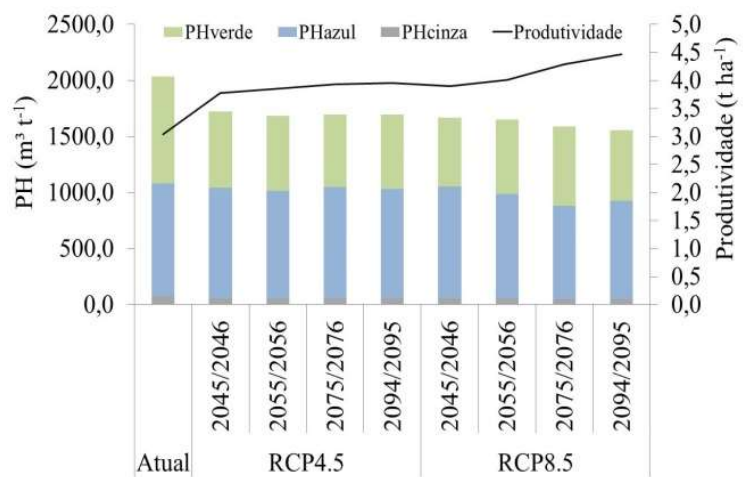

c)

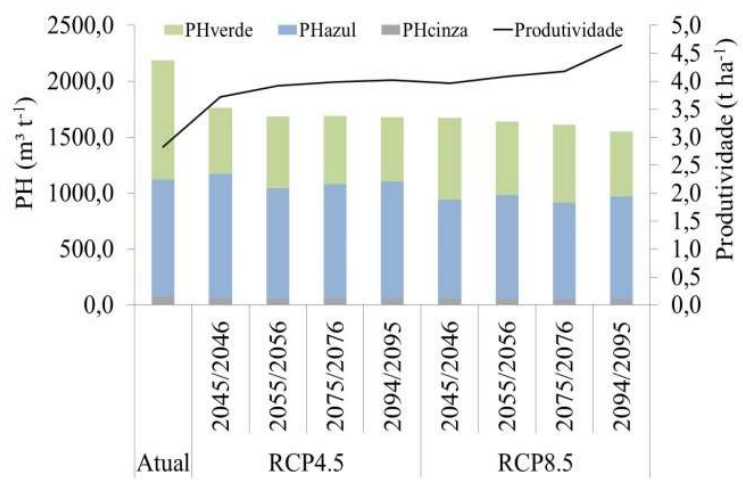

b)

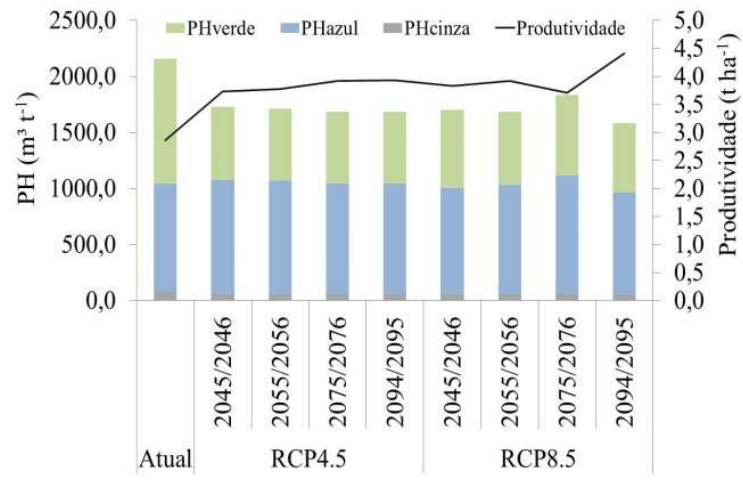

d)

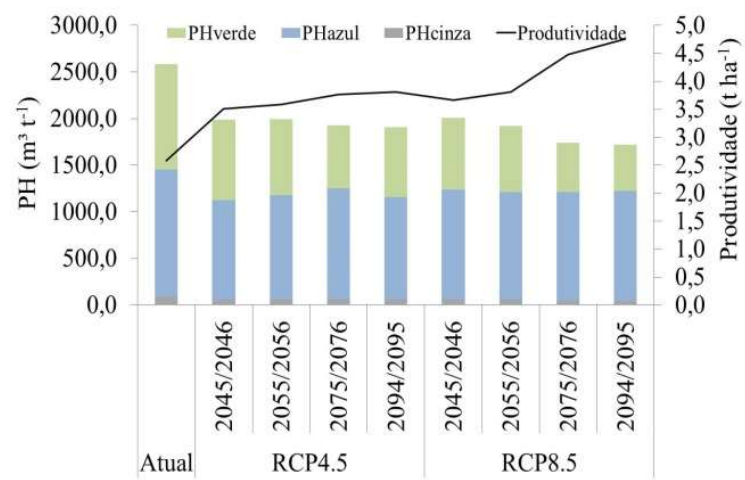

Figura 5. Estimativa da $\mathrm{PH}_{\text {soja }}$ azul, verde e cinza (colunas) e a produtividade total (linhas) para as cultivar de soja TMG 1288RR, projetada pelos modelos MIROC5 para o cenário de RCP 4.5 e RCP 8.5, para os três períodos: atual (utilizado como referência), médio prazo (2045/2046 e 2055/2056) e longo prazo (2075/2076 e 2094/2095) para os municípios de Balsas - MA (a), Campos Lindos- TO (b), Baixa Grande do Ribeiro - PI (c) e Formosa do Rio Preto -BA (d). 
Analisando a $\mathrm{PH}_{\text {soja }}$ por contribuição percentual de cada componente (azul, verde e cinza), identifica-se que as componentes sofreram grande variação percentual até o final desse século. Verifica-se que a componente azul da $\mathrm{PH}_{\text {soja, }}$ gerada pelos modelos HadGEM2-ES (Tabela 2) e MIROC5 (Tabela 3), sob as RCP 4.5 e 8.5, sofrerá aumento da sua contribuição, em praticamente todos os municípios, com exceção de Formosa do Rio Preto - BA. Porém nesse município as projeções geradas pelo HadGEM2-ES, sob a RCP 4.5, indicam aumento da componente verde da $\mathrm{PH}_{\text {soja }}$ a médio e a longo prazo. Os decréscimos mais acentuados da componente verde gerados pelo HadGEM2-ES, sob a RCP 4.5, foram para Campos Lindos TO (33\%) e Formosa do Rio do Preto - BA (31\%). As projeções geradas pelo MIROC5, em ambos os cenários, também apresentaram valores decrescentes, com destaque Baixa Grande do Ribeiro - PI (34\%), sob a RCP 4.5, e Formosa do Rio do Preto - BA (29\%), sob a RCP 8.5. As contribuições percentuais da componente cinza da $\mathrm{PH}_{\text {soja, }}$ para ambos modelos e cenários de RCP, sofreram pouca variação ao logo do período, porque adotou-se taxa de aplicação por hectare dos agroquímicos no campo e fração de lixiviação/escoamento praticamente constante ao longo dos anos em todos os quatro municípios.

$\mathrm{Na}$ atualidade para se produzir $1 \mathrm{~kg}$ de soja, em média, para os quatro municípios que representam a região Matopiba, são necessários 2.252,0 litros de água. Sendo que 47,6\% (1067,5 litros) dessa água corresponde a componente verde da $\mathrm{PH}_{\text {soja }}$, 49\% (1036,2 litros) a

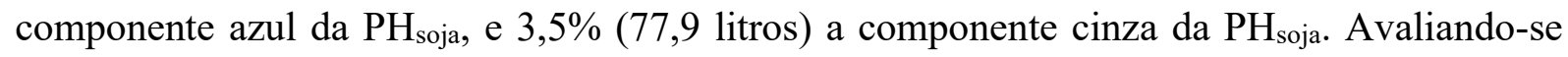
individualmente cada município, observa-se que Formosa do Rio Preto - BA, que tem a menor

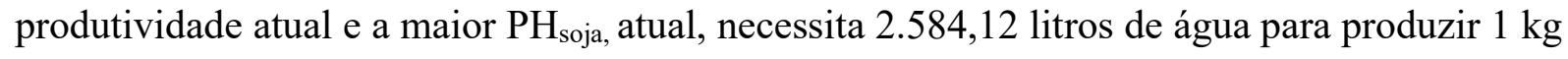
de grãos de soja, sendo que 52,8\% (1.364,4 litros) dessa é água azul, 43,9\% (1.108,6 litros) água verde, e 3,3\% (85,2 litros) água cinza. Em Balsas - MA, que tem a maior produtividade atual e a menor $\mathrm{PH}_{\text {soja }}$ atual, são necessários 2.036,6 litros de água para produzir $1 \mathrm{~kg}$ de soja, onde 46,9\% (955,7 litros) dessa água é verde, 49,5\% (1.008,5 litros) azul e 3,6\% (72,4 litros) cinza. Campos Lindos - TO, com a terceira maior produtividade e $\mathrm{PH}_{\text {soj }}$ atual, necessita de 2.158,9 litros de água para produzir $1 \mathrm{~kg}$ de soja, sendo que 51,6\% (1.114,0 litros) é água verde, 44,8\% (967,2 litros) azul, e 3,6\% (76,9 litros) cinza. No município de Baixa Grande do Ribeiro - PI, terceira maior produtividade atual e segunda maior $\mathrm{PH}_{\text {soja }}$ atual, usa-se 2.187, 7 litros de água para a produção de $1 \mathrm{~kg}$ de soja, onde 48,7\% (1.066,3 litros) é água verde, 47,7\% (1.043,7 litros) azul e 3,6\% (77,7 litros) cinza. 
Esses valores de $\mathrm{PH}_{\text {soja, }}$ encontrados para os quatro municípios maiores produtores de soja da região do Matopiba estão de acordo com os encontrados na literatura. A exemplo de Hoekstra e Chapagain (2008) e Mekonnen e Hoesktra (2011), que ao analisarem a média global da $\mathrm{PH}_{\text {soja }}$ encontram valores de $1.958 \mathrm{~m}^{3} \mathrm{t}^{-1}$ e $2.145 \mathrm{~m}^{3} \mathrm{t}^{-1}$, respectivamente. Mekonnen e Hoesktra (2011) encontraram para o Brasil uma $\mathrm{PH}_{\text {soja }}$ total de 2.202,0 $\mathrm{m}^{3} \mathrm{t}^{-1}$. Arena et al. (2011) encontraram uma $\mathrm{PH}_{\text {soja }}$ total na Argentina de 2.440,7 $\mathrm{m}^{3} \mathrm{t}^{-1}$ para cultivos não irrigados e de 2.572,2 $\mathrm{m}^{3} \mathrm{t}^{-1}$ para cultura irrigada. Ercin et al. (2012) encontraram para cultivo não irrigado uma $\mathrm{PH}_{\text {soja }}$ total de 2.651,0 $\mathrm{m}^{3} \mathrm{t}^{-1}$ na França, e de $3.172 \mathrm{~m}^{3} \mathrm{t}^{-1}$ no Canadá. Bleninger e Kotsuka (2015), para Maringa - PR, encontraram $\mathrm{PH}_{\text {soja }}$ total de $2.210 \mathrm{~m}^{3} \mathrm{t}^{-1}$. Costa et al. (2014) encontrou para a Paragominas a $\mathrm{PH}_{\text {soja }}$ total de $1.306 \mathrm{~m}^{3}$ ton $^{-1}$.

As produtividade de soja obtida sob cenários de mudanças climáticas, sugerem que a cultivar de soja TMG 1288 RR se favorecerá sobre essas condições climáticas. Nunes et al. (2016) e Silva et al. (2018), verificaram que a cultivar de soja TMG 1288 RR apresenta boa resistência ao estresse hídrico. Apesar das projeções indicarem aumento da temperatura e diminuição da precipitação, até o final desse século na região do Matopiba, os acumulados de precipitação ainda atenderão a demanda hídrica da soja, que varia de 450 a $850 \mathrm{~mm}$, o que também pode justificar esse aumento observado na produtividade futura da soja. Além da precipitação e temperatura, outro fator que pode ter influenciado para o aumento da produtividade futura da soja, é a concentração de $\mathrm{CO}_{2}$, que sob cenários de mudanças climáticas apresentam valores elevados. Boote et al. (2011) observaram que a soja responde positivamente ao aumento associado da temperatura do ar com o da concentração de $\mathrm{CO}_{2}$ na atmosfera, devido aos efeitos diretos de fertilização de $\mathrm{CO}_{2}$ na soja. Castillo (2016), ao analisar os efeitos das mudanças da temperatura, precipitação e concentração de $\mathrm{CO}_{2}$ na atmosfera, sobre a demanda de água e o rendimento de grãos da soja na bacia do rio Potiribu - RS verificou incremento da produtividade em comparação com a média histórica e atribuiu essa condição principalmente ao aumento da concentração atmosférica de $\mathrm{CO}_{2}$. Abreha et al. (2012), ao investigar o impacto de um clima hipotético e cenários de $\mathrm{CO}_{2}$ na América do Sul, verificaram que o efeito combinado da duplicação de $\mathrm{CO}_{2}$, aumento de $2^{\circ} \mathrm{C}$ na temperatura do ar e $10 \%$ na precipitação, refletindo no aumento da produção do milho, que também é uma planta C3. Sun et al. (2012), em estudo sobre a mudanças climáticas na China sob condição de irrigação, verificaram uma tendência de queda na evapotranspiração da cultura e o aumento exigência de água para irrigação devido a fatores climáticos. 
Assim como foi encontrado nessa pesquisa, identificou-se diminuição da evapotranspiração de cultura e o aumento de produtividade, o que resultou em redução da PH total. Bocchiola et al. (2013), ao estudarem a PH do milho sobre condições mudança climáticas na Itália, encontraram resultados que evidenciam claramente que é provável que a demanda de água azul atinja seu pico no futuro. Esse estudo também indicou que o uso relativo da água se tornou muito alto e a demanda de água para irrigação foi de até $230 \%$. Os resultados também podem ser comparados com outro estudo de Bocchiola (2015), onde se verificou que o rendimento do arroz é afetado negativamente quando a temperatura aumenta, a $\mathrm{PH}_{\text {azul }}$ necessária para atender a demanda de evapotranspiração encontrado foi maior para a altas temperaturas e quando ao mesmo tempo a precipitação é baixa, são necessárias grandes quantidades de água azul para inundações durante a estação de cultivo. Shrestha et al. (2017), em estudo para quantificar a PH da produção de arroz na Tailândia sobre condições irrigadas e de sequeiro, verificaram que tanto a evapotranspiração da cultura quanto a produtividade aumentaram, e consequentemente, observou-se uma diminuição significativa da $\mathrm{PH}$ para os períodos futuros.

As projeções futuras indicam que, em média, para produzir $1 \mathrm{~kg}$ de soja em Balsas MA, Campos Lindo - TO e Baixa Grande do Ribeiro - PI serão necessários de 1600 a 1700 litros de água. Esses valores, quando comparado a condição atual, apontam para redução de 500 litros na produção desse grão. Em Formosa do Rio Preto - Ba as projeções indicam que é necessário um pouco mais de água, 1800 litros para produção de $1 \mathrm{~kg}$ de soja. Quando comparada essa condição futura a atual, verifica-se redução de mais de 700 litros para a produção de soja. Entretanto, apesar das projeções, sob cenários de mudanças climáticas, apontarem redução da $\mathrm{PH}_{\text {soja }}$ na região do Matopiba, como mostra as Tabelas 2 e 3, as componentes apresentaram comportamento oposto. Sob cenários de mudanças climáticas a componente azul da $\mathrm{PH}_{\text {soja }}$ aumenta e componente verde diminui na maioria dos municípios. Assim, como a disponibilidade de água verde diminuirá, será necessário um maior volume de água azul para manter produtividade da soja elevada, e umas das alternativas será o cultivo irrigado, o que pode aumentar ainda mais a contribuição da componente azul. Esses resultados são compatíveis ao encontrados por Bacchiola et al (2013) e Bacchiola (2015) na Itália, Papadopoulou et al. (2015) na Grécia, e Shrestha et al. (2017) na Tailândia. As projeções de aumento da demanda pela água azul, indica que o uso apenas do valor do total da $\mathrm{PH}_{\text {soja não }}$ fornece a análise do impacto da PH sob cenários de mudanças climáticas. Uma vez que, nesse estudo, fica claro que, apesar da diminuição futura da $\mathrm{PH}_{\text {soja }}$, o aumento da componente azul poderá resultar em impactos significativos sobre os recursos hídricos da região do Matopiba. 
Tabela 3. Contribuição percentual das componentes verde, azul e cinza da PHsoja, atual (referência) e projetada pelo modelo MIROC5, para os cenários de RCP 4.5 e 8.5 e os período a médio prazo (2045/2046 e 2055/2056) e longo prazo (2075/2076 e 2094/2095), para os municípios de Balsas - MA, Campos Lindos- TO, Baixa Grande do Ribeiro - PI e Formosa do Rio Preto -BA.

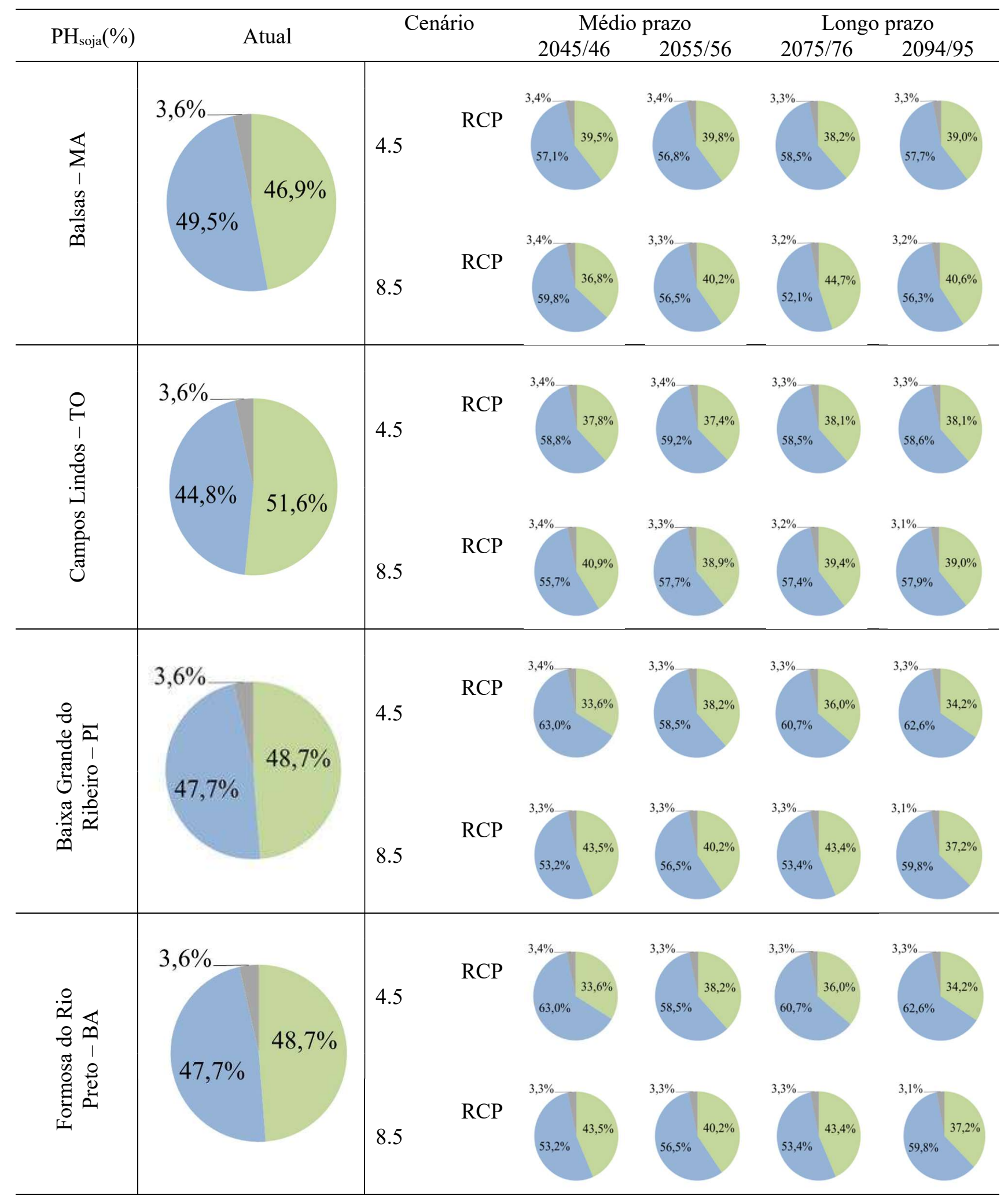


A $\mathrm{PH}_{\text {cinza }}$ pouco influenciou na $\mathrm{PH}_{\text {total }}$ dos sistemas produtivos de grãos da região. Apesar do comportamento decrescente da $\mathrm{PH}_{\text {cinza }}$ futura, é provável que a componente cinza aumente ao longo dos cenários de mudanças climáticas, devido as simulações levarem em consideração apenas o volume de assimilação de fertilizantes nitrogenados, necessários para uma alta produtividade, deixando de lado os poluentes relevantes, como: fósforo e os pesticidas.

\section{CONCLUSÕES}

Os resultados obtidos nesse estudo indicam que as projeções da produtividade e $\mathrm{PH}_{\text {soja, }}$, são altamente dependentes dos cenários climáticos considerados. Sob cenários de mudanças climáticas a produtividade da soja aumentará em até 2,0 tha ${ }^{-1}$ e a $\mathrm{PH}_{\text {soja }}$ diminuirá em até 700 $\mathrm{m}^{3} \mathrm{t}^{-1}$, em relação ao período atual, para todos os quatro municípios. Indicando que a quantidade de água total utilizada durante o processo de cultivo da soja diminuirá. Por outro lado, sob cenário de mudanças climáticas, a demanda por água azul aumentará em relação a água verde. Isso porque a componente verde da $\mathrm{PH}_{\text {soja }}$ decrescerá na proporção que aumentará a

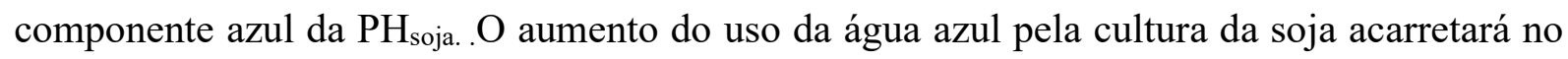
aumento da irrigação, para manter bom nível de produtividade, provocando maior pressão sobre os recursos hídricos da região do Matopiba. No caso da componente cinza da $\mathrm{PH}_{\text {soja, deve se }}$ ponderar que essa componente aumentará sob cenários de mudanças climáticas, uma vez que neste estudo foi avaliado apenas os fertilizantes nitrogenados e com valores praticamente constantes. Diante desses cenários de mudanças climáticas encontrados, sugere-se que nessa região sejam adotadas práticas agrícolas que reduzam ou mantenham a $\mathrm{PH}_{\text {soja }}$ em níveis aceitáveis. Esses resultados também evidenciam a necessidade do gerenciamento dos recursos hídricos na região do Matopiba.

\section{REFERÊNCIA BIBLIOGRÁFICA}

Abrha, B., Delbecque, N., Raes, D., Tsegay, A., Todorovic, M., Heng, L., Vanutrecht, E., Geerts, S., Garcia-Vila, M., Deckers, S. Sowing strategies for barley (hordeum vulgare 1.) based on modelled yield response to water with Aquacrop. Experimental Agriculture, 48(2), 252-271. Doi: https://doi.org/10.1017/S0014479712000166

Arena, A. P., Piastrellini, R., Civit, B. Water Footprint of soybean production in Argentina. Life Cycle Management Conference, Berlim, Alemanha, 2011. 
Bellouin, N., Rae, J., Jones, A., Johnson, C., Haywood, J., Boucher, O. Aerosol forcing in the Climate Model Intercomparison Project (CMIP5) simulations by HadGEM2-ES and the role of ammonium nitrate. Journal of Geophysical Research, 116(d20). Doi: https://doi.org/10.1029/2011JD016074

Bleninger, T., Kotsuka, L. K. Conceitos de água virtual e Pegada Hídrica: Estudo de caso da soja e óleo de soja no Brasil. Revista Recursos Hídricos, 36(1), 15-24. Doi: https://10.5894/rh36n1-2

Bocchiola, D., Nana, E., Soncini, A. Impact of climate change scenarios on crop yield and water footprint of maize in the Po valley of Italy. Agricultural Water Management, 116, 50-61.Doi: https://doi.org/10.1016/j.agwat.2012.10.009

Bocchiola, D. Impact of potential climate change on crop yield and water footprint of rice in the Po valley of Italy. Agricultural Systems, 139, 223-237. Doi: https://doi.org/10.1016/j.agsy.2015.07.009

Chapagain, A. K.; Hoekstra, A. Y.; Savenije, H. H. G.; Gautam, R. The water footprint of cotton consumption: an assessment of the impact of worldwide consumption of cotton products on the water resources in the cotton producing countries. Ecological Economics, 60(1), 186-203. Doi: https://doi.org/10.1016/j.ecolecon.2005.11.027

Castillo, A. C. Efeito das mudanças climátias sobre a demanda de água para irrigação e o rendimento de grãos da cultura a soja na bacia do Rio Potiribu, Rio Grande do Sul. Porto Alegre: UFRGS. 2016. Tese de Doutorado.

Costa, D. C., Martorano, L. G., Marques, M. C., Lisboa, L. S., Barbosa, P. S. P., Barbosa, A. M. S. Estimativa da Pegada Hídrica Cinza no controle da Lagarta-da-soja (Anticarsia gemmatalis), no polo de produção de grãos Paragominas, Pará. In: Anais... XVIII Congresso Brasileiro de Meteorologia, Recife, 2014.

Ercin, A.E., Hoekstra, A.Y. Carbon and Water Footprints: Concepts, methodologies and policy responses. World Water Assessment Programme, Side Publications Series, n.4, UNESCO, Paris. 2012. 
Fader, M., Gerten, D., Thammer, M., Lotze-Campen, H., Lucht, W., Cramer, W. Internal and external green-blue agricultural water footprints of nations and related water and land savings through trade. Hydrology and Earth System Science, 15(5), 1641-1660. Doi: https://doi.org/10.5194/hess-15-1641-2011

Habtemariam, L.T., Kassa, G. A., Gandorfer, M. Impact of climate change on farms in smallholder farming systems: Yield impacts, economic implications and distributional effects. Agricultural Systems, 152, 58-56. Doi: https://doi.org/10.1016/j.agsy.2016.12.006

Hargreaves, G. H., Samani, Z. A. Reference crop evapotranspiration from temperature. Applied Engineering Agriculture, 1(2), 96-99. Doi: https://doi: 10.13031 / 2013.26773 Hoekstra, A.Y., Hung, P.Q. Virtual water trade: A quantification of virtual water flows between nations in relation to international crop trade. Value of Water Research Report Series $\mathrm{n}^{\circ} 11,2002$.

Hoesktra, A. Y. Virtual water trade: Proceedings of the International Expert Meeting on Virtual Water Trade. Value of Water Research Report Series n $12,2003$.

Hoekstra, A. Y., Chapagain, A. K. Globalization of Water: Sharing the Planet's Freshwater Resources. Blackwell Publishing, Oxford, UK, 2008

Hoekstra, A. Y., Chapagain, A. K., Aldaya, M. M., Mekonnen, M. M. (2011) The water footprint assessment manual. 1.ed., London.

Jones, P. G., Thornton, P. K. Generating downscaled weather data from a suite of climate models for agricultural modelling applications. Agricultural Systems, 114, 1-5, Doi: https://doi.org/10.1016/j.agsy.2012.08.00

Lamond, R. E., Wesley, T. L. Adubação nitrogenada no momento certo para soja de alta produtividade. Informações Agronômicas, 95, 6-7, 2001.

Nunes, A. C., Bezerra, F. M. L., Silva, R. S., Silva Júnior, J. L. C., Gonçalves, F. B., Santos, G. A. Agronomic aspects of soybean plants subjected to deficit irrigation. Revista Brasileira de Engenharia Agrícola e Ambiental, 20, 654-659. Doi: https://doi.org/10.1590/1807-1929/agriambi.v20n7p654-659

Mekonnen, M. M., Hoekstra, A.Y. The green, blue and grey water footprint of crops and derived crop products. Hydrology and Earth System Sciences, 15, 1577-1600. Doi: https://doi.org/10.5194/hessd-8-763-2011 
Miranda, E. E. D., Magalhaes, L. A., Carvalho, C. A. D. Proposta de Delimitação Territorial do MATOPIBA. EMBRAPA: 1-18 p. 2014.

Papadopouloua, M.P., Charchousib, D., Tsoukalab,V. K., Giannakopoulosc, C., Petrakis, M. Water footprint assessment considering climate change effects on future agricultural production in Mediterranean region. Desalination and Water Treatment, 57(5), 1-11. Doi: https://doi.org/10.1080/19443994.2015.1049408

Rolla, A. L., Nuñez, M. N., Guevara, E. R., Meira, S. G., Rodriguez, G. R., Ortiz de Zárate, M. I. Climate impacts on crop yields in Central Argentina. Adaptation strategies. Agricultural Systems ,160, 44-59. Doi: https://doi.org/10.1016/j.agsy.2017.08.007

Rost, S., Gerten, D., Bondeau, A., Lucht, W., Rohwrer, J. Agricultural green and blue water consumption and its influence on the global water system. Water Resources Research, 44(9), .Doi: https://doi.org/10.1029/2007WR006331

Shrestha, S., Chapagain, R., Babel, M. S. Quantifying the impact of climate change on crop yield and water footprint of rice in the Nam Oon Irrigation Project, Thailand. Science of the Total Environment, 599-600, 689-699. Doi: https://doi.org/10.1016/j.scitotenv.2017.05.028

Silva, V. P. R., Silva, R. S., Maciel, G. F., Braga, C. C., Silva Júnior, J. L. C., Souza, E. P., Almeida, R. S. R., Silva, M. T., Holanda, R. M. Calibration and validation of the AquaCrop model for the soybean crop grown under different levels of irrigation in the Motopiba region, Brazil. Ciência Rural, 48(01). Doi: https://doi.org/10.1590/0103$8478 \mathrm{cr} 20161118$

Souza, P. J. O. P., Ortega-Farias, S., Rocha, E. J. P., Sousa, A. M. L., Souza, E. B. Consumo hídrico de soja no nordeste da Paraense. Irriga, 23(especial), 218-231. Doi: https://doi.org/10.15809/irriga.2016v1n01p218-231

Sun, S. K.,Wu, P. T.,Wang, Y. B., Zhao, X. N. Impacts of climate change on water footprint of spring wheat production: the case of an irrigation district in China. Spanish Journal of Agricultural Research, 10(4), 1176-1187. Doi: https://doi.org.10.5424/sjar/20121043004

Tongson, E. E., Alejo, L. A., Balderama, O. F. Simulating impacts of El Niño and climate change on corn yield in Isabela, Philippines. Climate, Disaster and Developmet Journal, 2(1), 29-39.Doi: https://doi.org/10.18783/cddj.v002.i01.a04 
Watanabe, S., Hajima, T., Sudo, K., Nagashima, T., Takemura, T., Okajima, H., Nozawa, T., Kawase, H., Abe, M., Yokohata, T., Ise, T., Sato, H., Kato, E., Takata, K., Emori, S., Kawamiya, M. MIROC-ESM 2010: model description and basic results of CMIP520c3m experiments. Geoscientific Model Development, 4, 845-872. Doi: https://doi.org/10.5194/gmd-4-845-2011 